\title{
Song Development: In Search of the Error-Signal
}

\author{
S. DERÉGNAUCOURT, ${ }^{a}$ P.P. MITRA, ${ }^{b}$ O. FEHÉR, ${ }^{a}$ K.K. MAUL,${ }^{a}$ T.J. LINTS, ${ }^{a}$ AND \\ O. TCHERNICHOVSKI ${ }^{a}$ \\ ${ }^{a}$ Department of Biology, City College, City University of New York, \\ New York, New York 10031, USA \\ ${ }^{b}$ Cold Spring Harbor Laboratories, Cold Spring Harbor, New York 11724, USA
}

\begin{abstract}
Song development provides an opportunity to study the mechanisms of vocal learning dynamically at molecular, cellular and systems levels, and across time scales ranging from minutes to months. To exploit these opportunities one needs to identify appropriate units, types and time scales of vocal change in nearly real time. The previous chapter by Tchernikovski $e$ t al. in this volume described techniques that make this research strategy feasible by allowing us to observe the song learning process through a "temporal microscope" with variable degrees of resolution. In this chapter we summarize some of the new observations and raise hypotheses about the learning strategy of the bird. We focus on inferences that can be drawn from behavioral observations to the nature and complexity of the instructive signal that guides the vocal change (error-signal). We examine two effects: i) the emergence of syllable types and ii) changes in features within a syllable type. We found that different features of the same syllable change during different and sometimes disjointed developmental windows. We discuss the possibility that song imitation is achieved by correcting partial errors, and that features of those partial errors change adaptively during development, perhaps concurrently with changes in perception and in motor proficiency. Those hypotheses can be best examined by across levels investigation, starting from identifying critical moments in song development and recording of articulatory dynamics and neural patterns when only a few features of specific syllables undergo rapid changes. Such investigation could relate behavioral events to brain mechanisms that guide song learning from moment-to-moment and across extended periods.
\end{abstract}

KEYWORDS: song development; vocal production; auditory perception

\section{INTRODUCTION}

Vocal learning proceeds in two (sometimes overlapping) phases. ${ }^{1}$ First, the bird memorizes a song it has heard (sensory learning) and then it modifies its vocal output

Address for correspondence: S. Derégnaucourt, Department of Biology, City College, City University of New York, 138th Street and Convent Avenue, New York, NY 10031. Voice: 212650-8608; fax: 212-650-8959.

sderegna@sci.ccny.cuny.edu; <http://ofer.sci.ccny.cuny.edu>

Ann. N.Y. Acad. Sci. 1016: 364-376 (2004). @ 2004 New York Academy of Sciences. doi: 10.1196/annals.1298.036 
to gradually approximate the remembered song model (sensorimotor learning). ${ }^{2}$ Some songbirds are age-limited learners, ${ }^{3}$ that is, vocal learning occurs more readily during specific stages of development. ${ }^{1,4}$ In the zebra finch, the sensory phase of the sensitive period occurs from day 25-65 post hatch and the sensory-motor sensitive period for song learning occurs between days 30-90..$^{5}$ Immelmann made careful observations of zebra finch song development and found that at about day 30, the young bird produces its first subsong, with sounds that appear vague, without a distinct frequency structure of the syllables. About two weeks later, the bird starts producing structured syllables (with identifiable spectral structure); some are already similar to syllables of the "tutor" bird, although, the order of syllables is not yet similar to that of the tutor song. Finally, between days 70-90, the order of syllable (song syntax) becomes stereotyped and the song motif fully crystallizes. Thereafter, the zebra finch rarely changes its song motif. ${ }^{5}$

Some features of song development appear to be relatively independent of sensory guidance. For example, birds that are kept socially isolated go through subsong and plastic song stages. ${ }^{6}$ Nevertheless, the timing and the outcome of song development are strongly affected by hearing, ${ }^{2,7}$ social factors, ${ }^{8}$ hormonal state ${ }^{9}$ and nutrition. ${ }^{10}$ Even the end of the sensitive period is not determined solely by age, but may be influenced by hormones and experience. ${ }^{11}$ For example, castration ${ }^{1}$ or withholding exposure to the song model ${ }^{12-14}$ can delay the closure of the sensitive period for song learning.

Until recently, it has been sufficient to describe song development in terms of qualitative stages as summarized above. However, recent observations of fine-tuned neural activity during song, including the description of a temporal code for song generation, ${ }^{15}$ the "rehearsal" of song during sleep, ${ }^{16}$ and the analysis of dynamic changes in auditory responses to songs ${ }^{17,18}$ require a correspondingly finely tuned analysis of the developmental process. For example, in the adult zebra finch, the activity of premotor HVC neurons resemble an array of accurate clocks, with each neuron "ticking" only once per song motif. ${ }^{15}$ Song-time is thought to be explicitly encoded by the sparse propagation of ticks across those neurons (as in a music box), but how does this clock function during song development? What happens to it when song syntax changes, or when motif duration gradually increases? Being able to identify (or even trigger) the appropriate vocal changes and then trace the progression of such changes during short time scales is a prerequisite for answering such questions and for understanding the role of HVC in song learning.

More generally, studying how the neural code evolves during vocal learning requires dynamic measurements of song development, identifying moment-to-moment changes in vocal activity and simultaneous monitoring of correlated neural signals. Furthermore, one of the most important open questions in vocal learning is how sensory-motor conversion is achieved, namely, what are the features of the instructive (error) signal that guides vocal changes toward the model? At the motor end, measurements of vocal changes and of the articulatory and neural patterns that give rise to those changes are essential, but not yet fully explored. In this chapter we discuss the problem of identifying momentary instances of vocal changes and of relating such events to the overall process of matching a song model. We attempt to relate this methodological problem to the nature of error correction (via auditory feedback). 

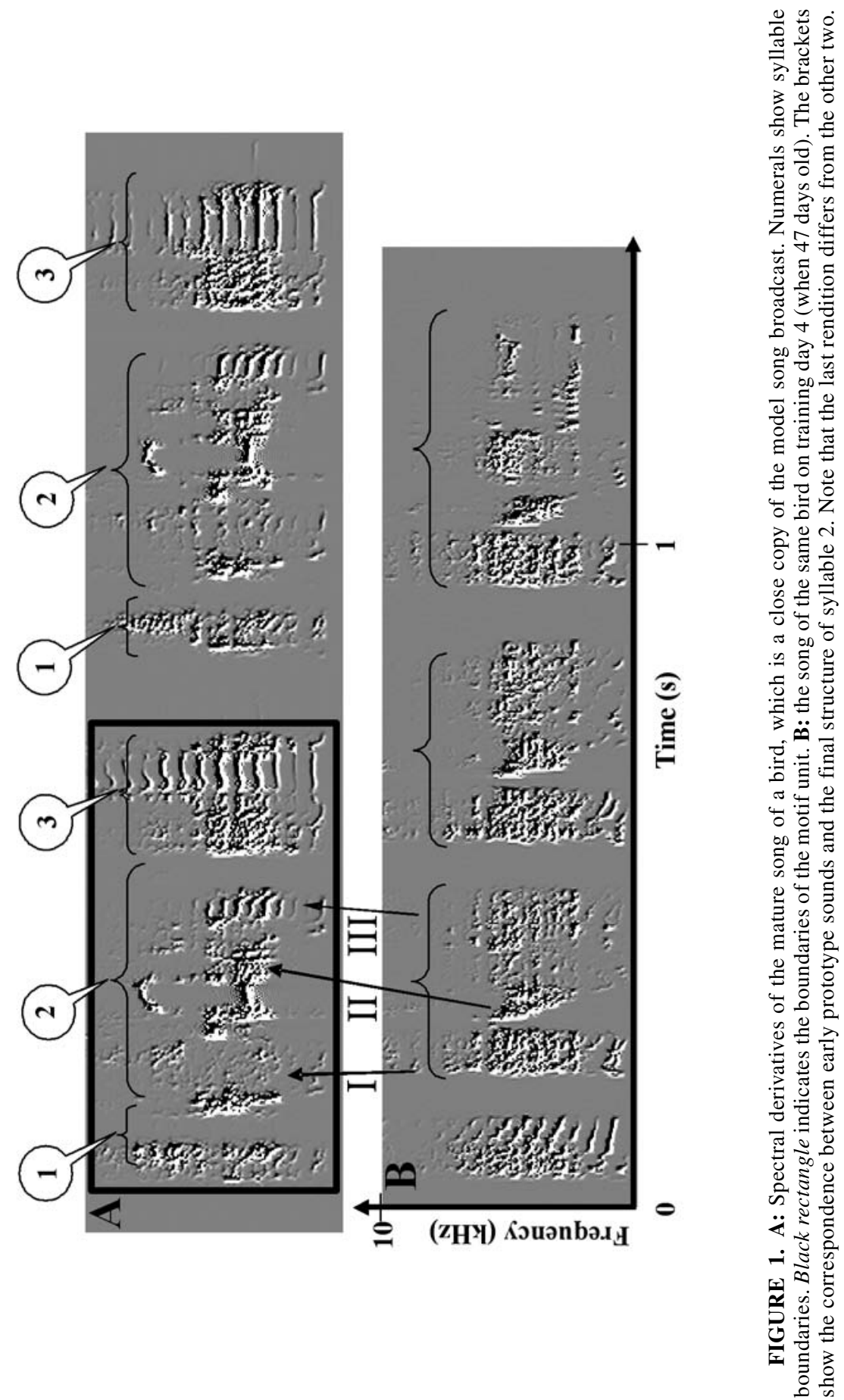


\section{EVALUATING THE ACOUSTIC ERROR}

During the sensory-motor phase of song learning the bird compares some memory of the song model to the auditory feedback of its own emerging song. Detection and reduction of acoustic differences can then be achieved, ${ }^{19,20}$ but what are the specific song features that are being compared?

FIGURE 1A presents the mature song (day 100 post hatch) of a bird that achieved a perfect copy of a song model. FIGURE 1B presents an earlier version of the song of the same bird when 47 days old, 4 days after the onset of training with song playbacks. ${ }^{21,22}$ As shown, the young bird already produces frequent back-to-back renditions of a statistically stable syllable type (we define "type" as an identifiable cluster in the distribution of syllable features, as elaborated in the methods chapter by Tchernichovski et al. in this volume). Tracking the imitation trajectory of this syllable from training day 4 to the conclusion of song development (as documented in the methods chapter) confirmed that it gave rise to a copy of model syllable 2. How can we estimate the acoustic error (in reference to the target syllable morphology) on training day 4 ? Comparing the prototype to the target, we can say that i) the first high-pitch sound of the song model is not present in the prototype (FIG. 1, arrow I), ii) the second high-pitch sound in the prototype (arrow II) is much too short with a down-sweep instead of a stable tone, iii) the last part of the prototype (arrow III) is still unstructured and iv) the duration of some of the prototypes is too short. We could continue to list mismatches, but is this useful? Do vocal changes occur by computing a global account of all acoustic differences between two complex sounds? Alternatively, are only "partial errors" corrected at any given time? If so, what could be the units, references, and features of such partial error estimates?

Obviously, it does not make sense to list mismatches arbitrarily as we did above, but experimentally we can examine what parts and what features of the song the bird changes at any given time during song development. Although we do not know how the features that we measure might correspond to those perceived and manipulated by the bird, we can hope that if the error-signal is indeed complex (e.g., including several partial error estimates), our features will capture some of this complexity, and detect natural time scales of different vocal changes that might be "hidden" in the imitation trajectory. Alternatively, if the error-signal is simple, we should not be able to detect a hidden structure in the developmental trajectory by looking at different features.

\section{TIME SCALES OF VOCAL CHANGES}

We now examine how different song features change during development, attempting to reveal the scope of vocal changes in song-time and in developmentaltime. ${ }^{23}$ We present vocal changes in units of daily change, but the same techniques can be used to explore finer time scales (down to several minutes).

The techniques of tracking clusters are described in the methods chapter (Tchernichovski et al., this volume). Briefly: we record the entire song development, partition the sound to syllable units and calculate simple features (such as mean pitch and frequency modulation) of each syllable. We then examine imitation trajectories by identifying clusters (types) of syllables and tracing each cluster back throughout 
A
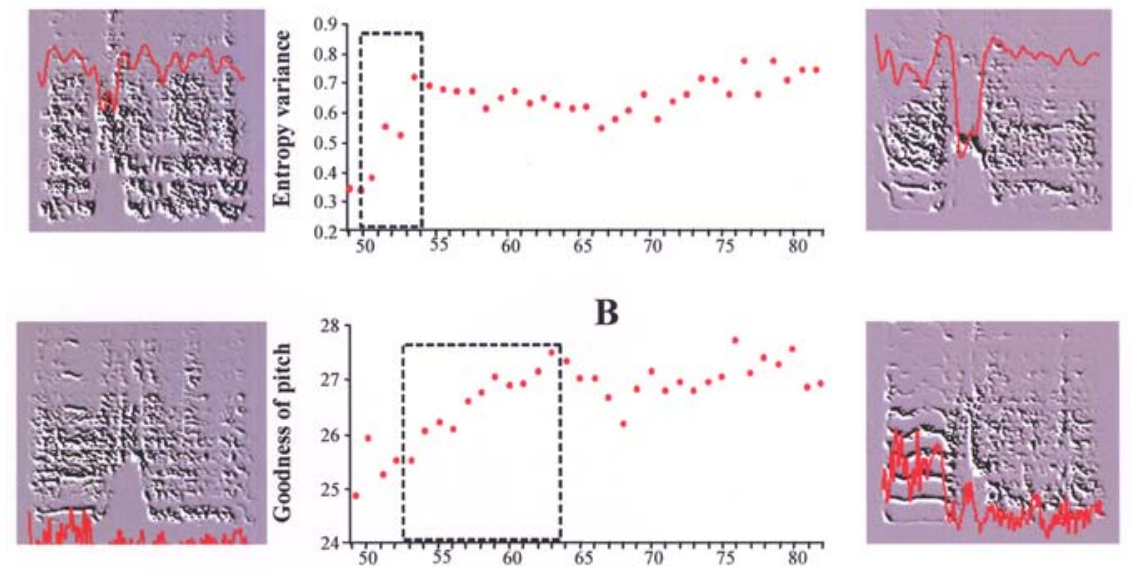

C
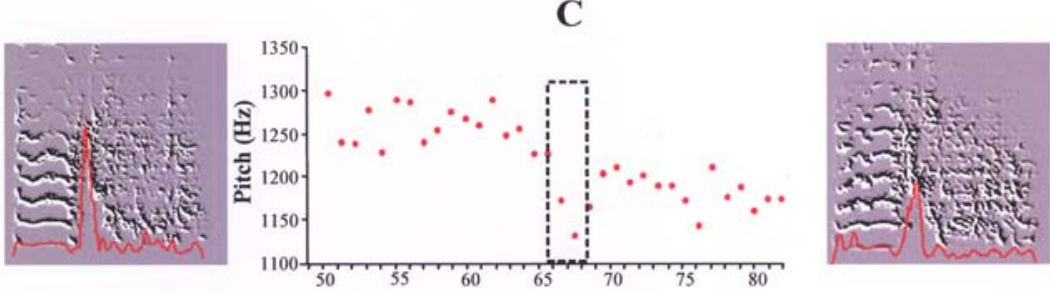

D
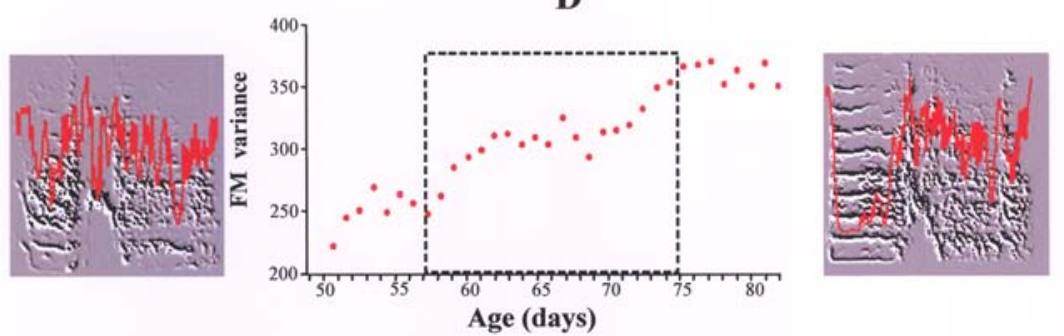

FIGURE 2. Time scales of vocal changes within one syllable type during song development: the middle panel show the mean daily value of syllable features. The dotted rectangle encloses the shortest developmental time that spans $80 \%$ of the overall change. Spectral derivatives of the syllable prior to change are shown on the left, and spectral derivatives of the syllable after the change are shown on the right. The curves show the actual values of the feature during a syllable. Note that each dot in the middle panel is an average obtained across all syllables produced during a day (typically 20-60 thousand). A: changes in Wiener entropy variance; B: changes of goodness of pitch; C: changes of pitch; D: changes of frequency modulation. (Color version of this figure appears online.) 
song development. All those steps are performed automatically, with the exception of cluster analysis, which is also done automatically but under visual supervision (for details see Sound Analysis Pro, User Manual <http://ofer.sci.ccny.cuny.edu>). We study the evolution of each cluster by plotting mean daily values of acoustic features. Those daily values are calculated by averaging all of the syllables of that cluster produced during each given day (usually ranging between 1,000-5,000). We can now examine the curve of feature values and find the shortest developmental window that spans a large portion (say 80\%) of the overall developmental change. This provides an estimate of the beginning and conclusion of a change in the value of a specific feature of a particular syllable.

FIGURE 2A (middle panel) presents daily values of a feature called "Wiener entropy variance". The dotted rectangle encloses the shortest interval that captures $80 \%$ of the change. As shown, the mean value of this feature increased rapidly between days 52-55 and changed little thereafter. How can we interpret this change? The variance of Wiener entropy captures the intra-syllabic transitions from tonal to broadband sounds. Such transitions often become more pronounced as the syllable matures. To see what exactly happened between days 52-55, we examine sonograms of this syllable just before the change, on day 51 (left panel), and compare those to sonograms obtained just after the change, on day 55 (right panel). As shown, the range (variance) of Wiener entropy increased because the first part of the syllable (particularly high-pitch note) became more tonal, causing a drop of Wiener entropy values only during the first part of this syllable.

FIGURE 2B shows how a different feature of the same syllable changes with time. Goodness of pitch captures the transition to harmonic frequency structure. The significant observation is that the time scale of this vocal change is very different and much longer (days 53-64) than that of Wiener entropy variance. Note also that the change can be seen throughout the syllable (the entire curve appears higher on the right panel). The next feature, pitch, shows rapid changes-but much later-from days 67-69 (FIG. 2C), whereas FM (FIG. 2D) changes very slowly (days 58-75).

Preliminary analysis of daily feature curves in 12 birds suggests to us that the example shown above is representative. Although the features we use are not orthogonal, we see in every single syllable a few uncorrelated curves that can be related to distinctive vocal changes such as time-warping, insertion of stop, ${ }^{23}$ perioddoubling $^{22}$ et cetera. Some of those changes are very rapid, whereas others are slow. Different vocal changes occur during different developmental times, and they are confined to specific parts of the song. That is, we observed no tight correlation between the occurrences of different vocal changes within a syllable, or between the occurrences of similar vocal changes across syllables.

Overall, our findings support the model of partial errors, but not conclusively. An additional question is whether different trajectories arise from a differential effect of motor development on the features that we measure. For example, as motor proficiency develops, the bird can better stabilize the frequency structure of harmonic stacks, but this should not indicate that something has changed in the error-signal. However, as a minimum, motor proficiency imposes constraints on the sounds that a bird can produce at a certain developmental stage. Behavioral observations alone cannot tell us if the error-signal succumbs to those constraints, but the ability to identify such events is only a first step, and a handle for further articulatory and brainlevel investigations of the sensory-motor conversion. 


\section{Zebra Finch}
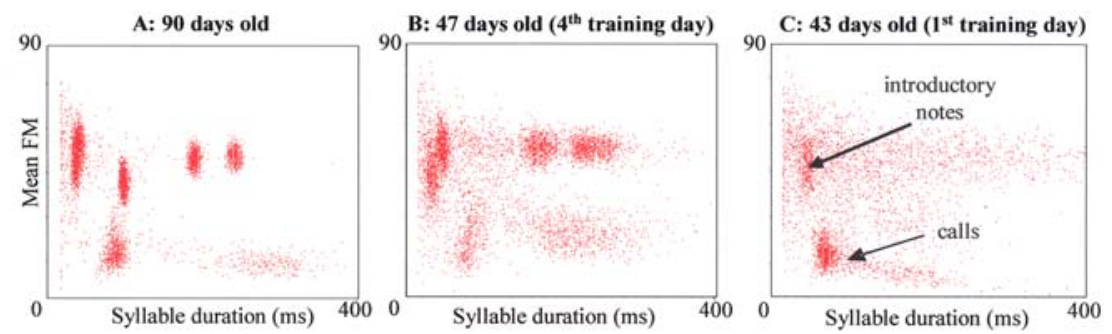

Human
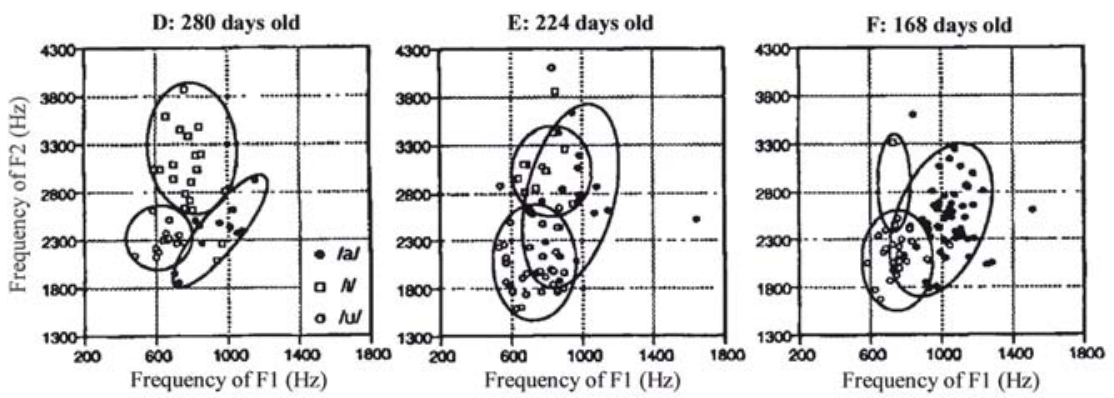

FIGURE 3. A-C: snapshots of DVD-maps [duration, FM] during song development. The production space of the subsong is continuous with only one or two vague clusters of simple calls, whereas song-like syllables do not form clusters. Within a few days after the onset of training, the production space takes the form of clusters. Note that even the very early clusters correspond to those of the mature song. D-F: categorical perception of speech in formant frequency space. (Reprinted with permission from Kuhl and Meltzoff. ${ }^{25}$ Copyright 1996, Acoustical Society of America.) The speech sounds fill up the space rather smoothly, but biases in perceptual sensitivity create virtual boundaries between categories. (Color version of this figure appears online.)

\section{PERCEPTUAL PLASTICITY AND MOTOR PROFICIENCY}

We started this chapter by asking how an early version of the song can be compared to the target song model (FIG. 1) and showed that song development can tell us the parts and features of the song that change during a specific "moment" of song development. We now ask more generally: what does it take for the bird to compare its own song (BOS) to the target song model during different developmental stages? In the example of BOS on day 4 (FIG. 1), the bird already produces repeated prototypes. Those prototypes are entities, on the production level, that the bird can potentially compare to the target. To quantify this notion, we plot the distribution of syllable features in the mature song (when a nearly-perfect model match has been achieved) and compare it to the distribution of syllable features on training day 4 (FIG. 3A-B). As shown, even the distribution of two features (duration versus FM) show robust clusters (or types) in the mature song (FIG. 3A). On training day 4 (FIG. 3B), we also see clusters, albeit sparser, and those clusters are clearly related to those 
of the mature song. We therefore conclude that on training day 4, it should be possible for the bird to compare features of its prototype syllable types to those of the target syllable types. However, this is no longer the case on training day 1 (FIG. 3C), on which BOS syllables are unstable and show no distinct types (except for calls), but sparse and continuous distribution. We only plot two features, but we see a similar effect across all features we can measure. Since aside from simple calls, we cannot detect any statistically stable clusters of sounds, we wonder how the bird can compare its sounds to those of the model.

In essence, what we see in FIGURE 3B-C is a transition from what has been traditionally called a subsong, to a plastic song structure. ${ }^{4}$ This transition has remarkable dynamics that can be observed in nearly real-time using Dynamic Vocal Development (DVD)-maps (see Tchernichovski et al., this volume), and we often see its progression on time scales of several minutes. The vast majority (and often all) of the clusters observed early on will evolve into copies of song-model syllables, that is, there are only few dead-ends. This observation is still preliminary, but it does suggest that the appearance of any one cluster indicates a significant commitment the bird is making during the very early stages of song learning, and that clusters (as entities) are not subject to major pruning. As noted, once the bird can produce sounds of distinct and stable types, it is easy to imagine how it may compare features of each type to those of model (template) syllables, but it is less clear how the bird can evaluate its performance prior to the emergence of distinct types.

Interestingly, changing motor patterns of vocal production for better approximation of an acoustic target is reminiscent of another example of vocal learning, that of speech learning and language emergence in humans. ${ }^{24}$ FIGURE 3D-F present developmental changes in the distribution of formant frequencies in human infants. ${ }^{25}$ As shown, both vocal learners, songbirds and humans, express a progression from generally unstructured, indistinct sounds to highly structured, acoustically complex, and motorically difficult sounds. ${ }^{24}$ Despite many gross differences between human speech and birdsong, in the crudest behavioral description, development of vocal production appears to follow a basic principle of organization, from unclustered to a clustered distribution. How can we explain this transition? In human speech, there are interesting correlates on both motor and perceptual levels: in the human infant, vocal production before 6-8 months is severely limited by maturation of the vocal tract and articulators, that is, physical and peripheral constraints restrict accurate production, but the difficulty of obtaining detailed recording in early stages of speech development limits the progress of research in this area. Much more is known at the perceptual level: in a nutshell, during the first year of life infants become perceptually "tuned" to their linguistic environment. ${ }^{26}$ The degree of coupling between dynamic changes in perceptions and production of speech is not yet known, but song development provides an opportunity to test, in a simple and more traceable system, how perceptual changes might guide vocal development as elaborated below.

The practicality of matching specific model syllables to the unstable and unclustered sounds of the subsong suggests that computing a complex error signal is not very useful at the very early stage of song learning. Indeed, even a while after global syllable features (first and second order statistics, as shown in the feature distribution map in FIG. 3) had stabilized, the fine-grained syllable morphology (as shown in the sonogram) is highly variable, suggesting that the early clusters are a very rough approximation of model sounds. We therefore suggest that for generating 


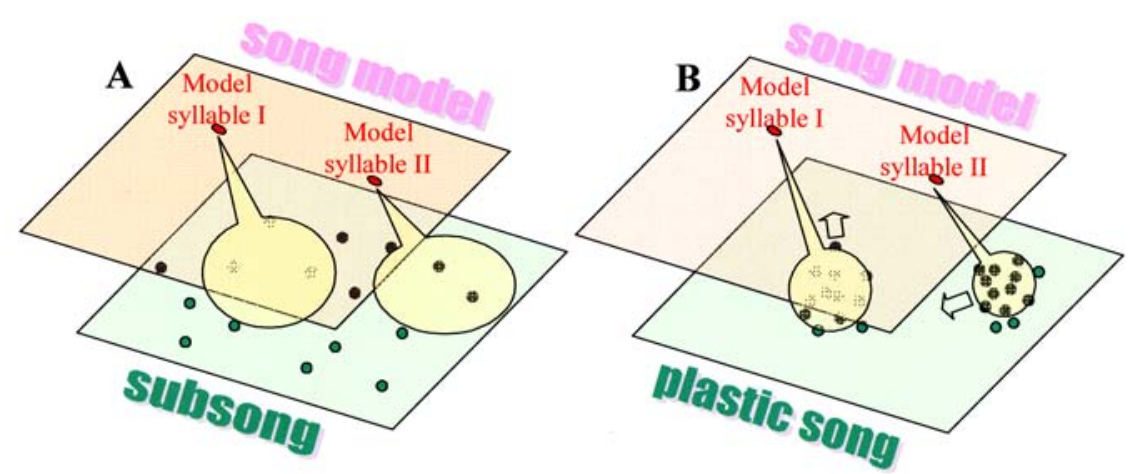

FIGURE 4. Comparing features of template sounds to features of the bird's own song during A: subsong stage where distribution of features is sparse and "search space" for even rough similarity must be broad, and B: during plastic song, where features of sounds that belong to distinct types can be compared to features of model syllables. The bird might then attribute error terms (arrows) for each cluster. Features are shown arbitrarily on a plane (we do not know what are the units and dimensionality of perceived sounds). Color version of this figure appears online.)

clusters, the bird needs to have broad perceptual tuning, perhaps centered on some global features (e.g., duration) of specific model sounds (FIG. 4A). Clusters can then emerge as a result of simple reinforcement learning, making sounds that are grossly similar to model syllables more likely. ${ }^{27,28}$ Once the bird established clusters, the perceptual requirements are likely to change (FIG. 4B): now the bird can start "moving" each cluster, using increasingly sharper perceptual tuning, to match the fine details of model syllable features.

Although FIGURE 4 is purely speculative, judging by overwhelming evidences from a variety of sensory-motor systems, ${ }^{29-31}$ it seems likely that development, ${ }^{32}$ auditory experience, ${ }^{33,34}$ and motor proficiency affect song perception, which can then affect the features of the error-signal. There is indirect evidence for perceptual plasticity during song learning: Nick has recently showed that auditory HVC activity is tuned to the song model in the awake bird, and to the BOS during sleep. ${ }^{35}$ Remarkably, the tuning to the BOS changes dynamically during development to match the current versions of BOS. ${ }^{17,18,36}$ Interesting clues for an effect of early experience on the metrics of song perception were recently obtained from EEG measurements of auditory evoked responses to playbacks of song syllables. Auditory evoked responses to song syllables have distinct patterns, those patterns vary across syllable types so that the pattern of the evoked response can tell us what syllable the bird has heard. Comparing patterns of evoked responses across birds showed that birds trained by the same tutor have similar auditory responses to playbacks of the same syllables, whereas birds trained by different tutors show much more variable responses to playbacks of the same sounds (those are all unfamiliar syllables). ${ }^{33,34}$ This finding suggests that early auditory experience has a major role in shaping auditory perception. It will be interesting to see if critical moments of song development, (e.g., when clusters emerge), is also the time when the effect of experience on auditory perception comes about. 


\section{MOTOR CONTROL AND SONG LEARNING}

The error signal is only useful to the extent that it can be converted to an appropriate motor action (articulatory gesture) that addresses the error. This is obviously an issue during the subsong stage (as we discussed above), but also later on, after the bird had achieved some motor proficiency. The bird's vocal organ (syrinx) is a complicated device, and when the bird performs a gentle articulatory gesture, it might experience abrupt and unexpected changes in sound structure. ${ }^{37}$ Such nonlinear effects might sustain beyond the boundaries of the sound that the bird attempts to change. ${ }^{38}$ The flip side of this argument is that a single articulatory gesture can, in principle, result in a series of sounds (just as throwing a ball may result in a series of events when the ball bounces on the floor). In other words, peripheral nonlinearities are a mixed blessing, allowing rapid transitions, sometimes at the cost of increasing control efforts. In the context of song learning, however, there is an additional problem that brings us back to the subject of perceptual tuning: if the bird hears two back-to-back sounds, it might be difficult for the bird to imitate them separately if productions mechanism of those two sounds are not distinct, namely, units of perception and units of production might be incompatible. We do not know what perceptual biases the bird might have prior to vocal experience, but as the bird acquires motor proficiency, it might be able to identify instances of nonlinearities in its own production mechanisms, and adjust its perceptual units accordingly. Evidence suggests that peripheral nonlinearities are taken into account during song learning. ${ }^{22}$

\section{VARIABILITY ACROSS TIME SCALES OF VOCAL LEARNING}

Early studies of song development showed that in contrast to the highly structured and stereotyped song of the adult bird, the young bird produces highly variable sounds. ${ }^{5}$ What is the role (or the consequences) of this variability on the vocal learning process? Based on variability observed in sonograms of early songs, it seems as if song development is a messy, erratic process. Observing song development by means of DVD-maps however, (see examples at <http://ofer.sci.ccny.cuny.edu>) gives the impression of a structured and smooth process. This contrast is due to different time scales of the two representations: the sonogram presents dynamics on millisecond time scale whereas a single frame of a DVD-map is a summary (distribution of first and second order statistics) of a few minutes of singing (about 5 orders of magnitude higher). The stability of the DVD-map is therefore an indication that the short-term variability in syllable morphology is constrained, and that song development is a structured process. The role of this short-term variability in song development is obscure, it might reflect the lack of subtle control on the vocal instrument or it could reflect a random-search strategy of guiding the imitation trajectory towards the target model. ${ }^{39}$ How can one distinguish between the two? If the variability is a mean of reaching a target, we would expect that once a target state is reached variability in the production of that sound should decrease abruptly. Since different features might reach their target during different developmental windows (as in FIG. 2), we should expect that the decrease of variability will correspond to those time scales. To the best of our knowledge, this hypothesis has not yet been tested. 


\section{SUMMARY}

We started by asking how we can estimate the acoustic error between the bird's current song and the song model (FIG. 1). To the extent that we can infer about the internal state from the actions the bird takes, measurements of song development suggest that the error-signal is neither simple nor stationary. Nevertheless, during any particular window of song development, we can identify distinct events of vocal change. Early events are the emergence of stable syllable types that are only roughly similar to some model sounds. Later events are changes in specific features of a particular syllable type. Some vocal changes progress rapidly, within hours or days, and other vocal changes progress slowly within weeks. Therefore, when attempting to interpret brain measures of a learning bird, one can distinguish between features of the acoustic error that the bird is currently engaged with, and other features of the same sound, that are currently "ignored" by the bird.

The technical feasibility of measuring song development should further encourage the interdisciplinary approach in the field of birdsong neurobiology. In particular, one should avoid reductionism when thinking about concepts such as errorsignal, since any view that ignores the roles of development, perception plasticity and motor proficiency is likely to be unrealistic (FIG. 5). Unfortunately, we do not

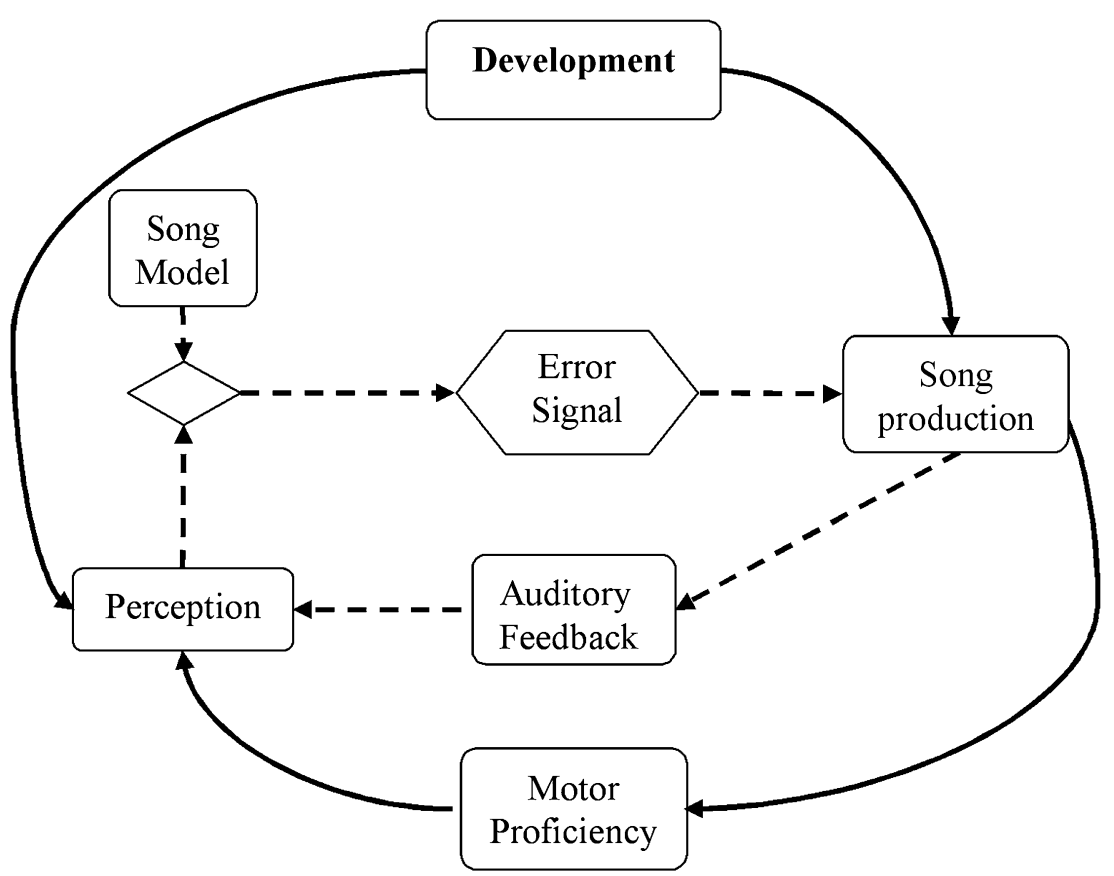

FIGURE 5. Perception plasticity and song learning. The dashed arrows show propagation of the feedback loop from moment-to-moment. Plain arrows show effects that occur on long time scales during development. 
have the means to judge what are the most difficult challenges that the birds encounter while mastering a song. If singing were like riding a bike, we could infer, after some observations, that balancing the bike is important, but it is much more difficult to appreciate (without ever riding a bike), the fundamental relations between speed and balance, and that it is the most proficient rider that can keep the bike balanced at a very low speed (indeed, learning to ride a bike can be very difficult at low speeds). By this metaphor we mean to say that interpreting brain activity during song learning should become much easier if we understood more about the nature of the task, and much can be achieved by performing perceptual or articulatory measurements during song learning, and by attempting to control the syrinx in-vitro so as to actually experience some of the challenges of song learning. Other non-acoustic factors such as social interactions can profoundly influence song learning. Measuring their contribution with the same accuracy as those achieved now with the mechanisms of vocal production is another challenge for the study of birdsong.

\section{ACKNOWLEDGMENTS}

Work reported here was supported by US Public Health Services (PHS) grant DC04722-04 to O.T., by Fondation Fyssen grant to S.D., and by the National Institutes of Health RCMI grant to CCNY.

\section{REFERENCES}

1. Noтteвонм, F. 1993. The search for neural mechanisms that define the sensitive period for song learning in birds. Neth. J. Zool. 43: 193-234.

2. KonISHI, M. 1965. The role of auditory feedback in the control of vocalization in the white-crowned sparrow. Z. Tierpsychol. 22: 770-783.

3. MARLER, P. 1970. A comparative approach to vocal learning song development in white-crowned sparrows. J. Comp. Physiol. Psychol. 71: 1-25.

4. Thorpe, W.H. 1961. Bird Song. Cambridge University Press. Cambridge, UK.

5. ImmelmanN, K. 1969. Song development in the zebra finch and other estrildid finches. In Bird Vocalizations. RA Hinde, Ed.: 61-74. Cambridge University Press. Cambridge, UK.

6. PrICE, P. 1979. Developmental determinants of structure in zebra finch song. J. Comp. Physiol. Psychol. 93: 260-277.

7. LeOnardo, A. \& M. Konishi. 1999. Decrystalization of adult birdsong by perturbation of auditory feedback. Nature 399: 466-470.

8. Kroodsma, D. \& R. Pickert. 1984. Sensitive phases for song learning: effects of social interaction and individual variation. Anim. Behav. 32: 389-394.

9. ARNOLD, A.P. 1975. The effects of castration on song development in zebra finches (Poephila guttata). J. Exp. Zool. 191: 261-278.

10. Nowicki, S., W.A. SeArCY \& S. Peters. 2002. Brain development, song learning and mate choice in birds: a review and experimental test of the "nutritional stress hypothesis". J Comp Physiol A. 188: 1003-1014.

11. Baptista, L.F. 1996. Nature and its nurturing in avian vocal development. In Ecology and Evolution of Acoustic Communication in Birds. D.E. Kroodsma \& E.H. Miller, Eds.: 39-60. Cornell University Press. Ithaca, NY.

12. EALES, L. 1985. Song learning in zebra finches: some effects of song model availability on what is learnt and when. Anim. Behav. 33: 1293-1300.

13. Morrison, R. \& F. NotTebohm. 1993. Role of a telencephalic nucleus in the delayed song learning of socially isolated zebra finches. J. Neurobiol. 24: 1045-1064. 
14. Jones, A., C. Ten CATE \& P.J.B. Slater. 1996. Early experience and plasticity of song in adult male zebra finches (Taenyopygia guttata). J. Comp. Psychol. 110: 354-369.

15. Hahnloser, R.H., A.A. Kozhevnikov \& M.S. Fee. 2002. An ultra-sparse code underlies the generation of neural sequences in a songbird. Nature 419: 65-70.

16. Dave, A.S. \& D. Margoliash. 2000. Song replay during sleep and computational rules for sensorimotor vocal learning. Science 290: 812-816.

17. Solis, M.M. \& A.J. Doupe.1999. Contributions of tutor and bird's own song experience to neural selectivity in the songbird anterior forebrain. J. Neurosci. 19: 4559-4584.

18. Mooney, R. 2000. Different sub threshold mechanisms underlie song-selectivity in identified HVc neurons of the zebra finch. J. Neurosci. 20: 5420-5436.

19. BRainaRd, M.S. \& A.J. Doupe. 2000. Interruption of a basal ganglia-forebrain circuit prevents plasticity of learned vocalizations. Nature 404: 762-766.

20. BRainard, M.S. \& A.J. Doupe. 2001. Post-learning consolidation of birdsong: stabilizing effects of age and anterior forebrain lesions. J. Neurosci. 21: 2501-2517.

21. AdRET, P. 1993. Operant conditioning, song learning and imprinting to taped song in the zebra finch. Anim. Behav. 46: 159-159.

22. Tchernichovski, O., P.P. Mitra, T. Lints \& F. Nottebohm. 2001. Dynamics of the vocal imitation process: how a zebra finch learns its song. Science 291: 2564-2569.

23. TCHERnichovski, O. \& P.P. Mitra. 2002. Towards quantification of vocal imitation in the zebra finch. J. Comp. Physiol. A 188: 867-878.

24. Doupe, A.S. \& P.K. KuHL. 1999. Birdsong and human speech: common themes and mechanisms. Annu. Rev. Neurosci. 22: 567-631.

25. KuHL, P.K, \& A.N. Meltzoff. 1996. Infant vocalizations in response to speech: vocal imitation and developmental change. J. Acoust. Soc. Am. 100: 2425-2438.

26. JuSCZYK, P.W. 1997. The Discovery of Spoken Language. MIT Press. Cambridge, MA.

27. Troyer, T.W. \& A.J. Doupe. 2000. An associational model of birdsong sensorimotor learning II. Temporal hierarchies and the learning of song sequence. J. Neurophysiol. 84: $1224-1239$.

28. Troyer, T.W. \& A.J. Doupe. 2000. An associational model of birdsong sensorimotor learning I. Efference copy and the learning of song syllables. J. Neurophysiol. 84:1204-1223.

29. Elbert, T., C. PAntev, C. Wienbruch, et al. 1995. Increased cortical representation of the fingers of the left hand in string players. Science 270: 305-307.

30. Mühlnickel, W., T. Elbert, E. TAUb \& H. Flor. 1998. Reorganization of auditory cortex in tinnitus. Proc. Natl. Acad. Sci. USA 95: 10340-10343.

31. KNUDSEN, E.I. 1999. Early experience and critical periods. In Fundamental Neuroscience. M.J. Zigmond, F.E. Bloom, S.C. Landis, et al., Eds.: 637-654. Academic Press. New York.

32. BRitTAn-Powell, E. F. \& R. J. Dooling. Development of auditory sensitivity in budgerigars (Melopsittacus undulatus). J. Acoust. Soc. Am. In press.

33. Espino, G. G., C. Lewis, D.B. Rosenfield \& S. A. HelekAr. 2003. Modulation of theta/alpha frequency profiles of slow auditory evoked responses in the songbird zebra finch. Neuroscience 122: 521-529.

34. Espino, G.G., D.B. Rosenfield \& S.A. Helekar. 2003. Similarities in neural correlates of song discrimination in zebra finches with similar song. Program No. 942.8. Abstract Viewer and Itinerary Planner. Society for Neuroscience. Washington, DC. Online.

35. NICK, T.A. 2003. Response bias: neural correlates of memory in the birdsong system. Program No. 294.2. 2003 Abstract Viewer and Itinerary Planner. Society for Neuroscience. Washington, DC. Online.

36. Ribeiro, S., G.A. Cecchi, M.O. Magnasco \& C.V. Mello. 1998. Toward a song code: evidence for a syllabic representation in the canary brain. Neuron 21: 359-371.

37. Fee, M.S., B. Shraiman, B. Pesaran \& P.P. Mitra. 1998. The role of nonlinear dynamics of the syrinx in the vocalizations of a songbird. Nature 395: 67-71.

38. Gardner, T, CECChi, M. Magnasco, et al. 2001. Simple motor gestures for birdsongs. Phys. Rev. Lett. 87: 208101.

39. Nelson, D.A. \& P. MARLER. 1994. Selection-based learning in bird song development. Proc. Natl Acad. Sci. U S A. 91: 10498-10501. 